\title{
Lésions moléculaires de la cystinurie
}

La cystinurie est une affection héréditaire, d'hérédité autosomique récessive, dont la fréquence varie de 1 pour 2500 chez les juifs originaires de Libye à 1 pour 15000 aux États-Unis. Elle est due à un défaut de transport rénal: la cystine, ainsi que les acides aminés basiques, ne sont pas réabsorbés; comme la cystine est très peu soluble, elle forme des calculs rénaux. La cystinurie fait partie des quatre maladies décrites comme "erreurs innées du métabolisme " en 1908 par Garrod. Elle est le prototype des maladies des transporteurs dont le mécanisme est longtemps resté, et est encore pour une part, mystérieux. En 1987, une protéine put être isolée par clonage d'expression dans les ovocytes de Xénope. Parmi les noms qui ont été donnés à cette glycoprotéine de $90 \mathrm{kDa}$, celui de rBAT prédomine : ce nom vient d'un système de transport dit $\mathrm{b}^{0++}\left(b^{0,+}\right.$ aminoacid transporter related) [1]. On a fait dès lors l'hypothèse [2] que la cystinurie pourrait être due à une anomalie de rBAT. Deux articles parus en avril 1994 dans Nature Genetics viennent à l'appui de cette hypothèse.

L'équipe dirigée par Kastner, associant des chercheurs du NIH, d'Israël et de Paris [3], a centré sa recherche sur la localisation du gène. A partir de dix-sept familles, dont six à fort taux de consanguinité, ils.ont démontré que le gène some 2. Ce gène, qu'ils appellent SLC3A1, est le même que rBAT.

Le travail de l'équipe hispanoitalienne dirigée par Palacin [4] visait à la démonstration directe du rôle joué par des anomalies de rBAT. Il est parti de la connaissance récente d'ADN complémentaires de plusieurs espèces, dont l'homme [2]. Les messagers correspondant à ces ADNc sont exprimés dans la bordure en brosse des tubules rénaux, mais aussi de l'intestin grêle. L'ADNc de $r B A T$ humain possède 2284 nucléotides et code pour une protéine de 663 acides aminés. Faisant appel à la " transcription illégitime", initialement décrite par une équipe française [5], on a utilisé pour ce travail des cellules lymphoblastoïdes. Après transcription inverse et amplification, on a employé la méthode de SSCP (single strand conformational polymorphism) [6]. En tout six mutations ont été observées, toutes de type fauxsens. Selon les malades, on les trouve sous forme d'homozygotes ou d'hétérozygotes composites. La plus fréquente s'est avérée une mutation Met $467 \rightarrow$ Thr, retrouvée chez six sujets non apparentés, alors que les cinq autres n'ont été observées qu'une fois. C'est donc une mutation Met $467 \rightarrow$ Thr qui a été choisie pour construire un rBAT anormal, dont on a injecté le messager au Xénope. L'anomalie abolissait presque complètement l'activité de transport de la L-cystine, de l'arginine mais aussi de la leucine*. Les travaux que nous venons de relater démontrent clairement le rôle des altérations de rBAT dans la genèse de la cystinurie. Ils n'en expliquent pas le mécanisme [7]. La protéine déduite de la séquence de l'ADNc apparaît peu hydrophobe et ne devrait traverser la membrane qu'une fois, alors que la plupart des protéines de transport clonées dans de multiples espèces la traversent douze fois. Le polypeptide rBAT ressemble à la sous-unité $\beta$ des pompes $\mathrm{Na}^{+} / \mathrm{K}^{+}$et $\mathrm{H}^{+} / \mathrm{K}^{+}$; elle a une extrémité NH2 cytoplasmique, une région transmembranaire et un volumineux segment glycosylé terminal extracellulaire. Il est à remarquer que cing des six mutations observées siègent dans la région C-terminale et non dans une zone transmembranaire. Une hypothèse possible [4] est que rBAT soit un constituant, peut-être régulateur, d'un transporteur complexe. C'est dire que la découverte d'anomalies de rBAT, pour intéressante qu'elle soit, pourrait ne pas épuiser la question de l'origine de la cystinurie. Des mutations ont été observées dans environ $30 \%$ des cas examinés [4]. D'autres lésions moléculaires pourraient exis-

* L'anomalie porte aussi sur les acides aminés neutres. Mais pour ceux-ci, il doit exister des mécanismes compensateurs car ils ne sont pas éliminés en excès dans les urines. 
ter dans le même gène ou d'autres voisins. Les méthodes génétiques, toutefois, n'ont pas, jusqu'à présent, révélé d'autres localisations chromosomiques.

On peut enfin remarquer qu'il s'agit probablement $\mathrm{du}$ premier gène identifié dans une affection héréditaire du transport d'un acide aminé. Dans un autre système, un défaut d'un cotransporteur $\mathrm{Na}^{+}$/glucose avait été décrit en 1991 [8] dans la malabsorption glucose/galactose.

J.-C. D.

1. Bertran J, Magnanin S, Werner A, Markovich $\mathrm{D}$, Biber J, Testar X, Zorzano A, Kühn I.C, Palacin M, Murer H. Stimulation of system $\mathrm{y}^{+}$-like aminoacid transport by the heavy chain of human 4F2 surface antigen in Xenopus laevis oocytes. Proc Natl Acad Sci USA 1992; 89 : 5606-10.

2. Lee WS, Wells RG, Sabbag RV, Mohandas TK, Hediger MA. Cloning and chromosomal localization of a human kidney cDNA involved in cystine, dibasic and neuural aminoacid transport. J Clin Invest 1993 ; 91 : 1959-63.

3. Pras E, Arber N, Aksentijevich I, Katz G, Schapiro JM, Prosen L, Gruberg L, Harel D, Liberman U, Weissenbach J, Pras $\mathrm{M}$, Kastner DL. Localization of a gene causing cystinuria to chromosome 2p. Nature Genet 1994; 6: 415-9.

4. Calonge MJ, Gasparini P, Chillaron J, Chillon M, Gallucci M, Rousaud F, Zelante L, Testar X, Dallapiccola B, Di Silverio F, Barcelo P, Estivill X, Zorzano A, Nunes V, Palacin M. Cystinuria caused by mutations in $\mathrm{rBAT}$, a gene involved in the transport of cystine. Nature Genet 1994; 6: 420-5. 5. Chelly J, Concordet JP, Kaplan, JC, Kahn A. Illegitimate transcription. Analysing any transcript in any cell-type. Proc Natl Acad Sci USA 1989; 86: 2617-21.

6. Dreyfus JC, Akli S, Poenaru L. Maladies de Tay-Sachs et de Sandhoff: les déficits en B-hexosaminidases, modèles de maladies des lysosomes. médecine/sciences $1992 ; 8$ : 797-804.

7. Wright EM. Cystinuria defect expresses itself. Nature Genet 1994; 6: 328-9.

8. Turk E, Zabel B, Mundios S, Dyer J, Wright EM. Glucose/Galactose malabsorption caused by a defect in the $\mathrm{Na}^{+} /$glucose cotransporter. Nature 1991; 350 : 354-6. 